\title{
Índice de Desempeño Integral ajustado a las localidades de Bogotá D.C.'
}

\author{
María del Pilar Sánchez Muñoz \\ Universidad de La Salle / Facultad de Ciencias Económicas y Sociales \\ Bogotá, Colombia \\ Daniel Fernando Lozano Toscano \\ Escuela Superior de Administración Pública / Facultad de Investigaciones \\ Bogotá, Colombia \\ Mauricio Fernando Moreno Infante \\ Alcaldía Municipal de San José de Cúcuta / Departamento Administrativo de Bienestar Social \\ Norte de Santander, Colombia
}

\begin{abstract}
En este documento se presenta un estudio de la descentralización fiscal y administrativa de las 19 localidades de Bogotá (Colombia), adaptando la metodología del Departamento Nacional de Planeación (DNP) para medir el desempeño integral de los entes territoriales, específicamente con los componentes de eficacia y gestión para el periodo 2009-12. Se encontró que el nivel de cumplimiento de metas en los planes de desarrollo de cada localidad es medio, a pesar de que existe una descentralización administrativa en las formas de desconcentración y delegación. La investigación también mostró que, según el índice de gestión, las localidades no tienen autonomía para llevar a cabo proyectos de inversión ni obtener recursos propios, debido a los lineamientos de la Alcaldía Mayor de la ciudad.
\end{abstract}

Palabras clave: presupuesto local; relaciones intergubernamentales; planeación económica regional.

Índice de Desempenho Integral aplicado às regiões administrativas locais de Bogotá D.C.

Neste artigo é apresentado um estudo da descentralização fiscal e administrativa de 19 regiões administrativas locais de Bogotá (Colômbia), adaptando-se a metodologia do Departamento Nacional de Planeación (DNP) para medir o desempenho integral das entidades territoriais, mais especificamente

DOI: http://dx.doi.org/10.1590/0034-7612141819

(C) (i)

Artículo recibido en 18 oct. 2014 y aprobado en 2 jun. 2016.

${ }^{1}$ El artículo es el resultado del trabajo de pasantía "Análisis de la descentralización fiscal y administrativa en las localidades de Bogotá del 2000 al 2012", realizado por Daniel Lozano y Mauricio Moreno para optar al título de Economista. Este trabajo fue dirigido por María del Pilar Sánchez Muñoz. Los autores participaron en la misma proporción durante todo el proceso de la investigación. 
os componentes de eficácia e gestão para o período de 2009-12. Encontrou-se que o nível de cumprimento das metas e dos planos de desenvolvimento de cada região administrativa local é médio, apesar da descentralização administrativa observada nas formas de delegação e desconcentração. A pesquisa também mostrou que, segundo o índice de gestão, as regiões administrativas locais não têm autonomia para levar a cabo projetos de investimento, nem obter recursos próprios, de acordo com as diretrizes da Prefeitura da cidade.

Palavras-chave: orçamento local; relações intergovernamentais; planejamento econômico geral.

A comprehensive performance index adjusted to the different districts of Bogotá D.C.

In this paper, we present a study of the fiscal and administrative decentralization that has occurred in the 19 districts of the City of Bogotá, Columbia, using and adapting the methodology of the National Department of Planning (Departamento Nacional de Planeación - DNP), to measure the comprehensive performance of these territorial entities, especially in terms of their management and efficacy in the period 2009-2012. The results of the study showed that level of success in achieving the targets contained in each district's development plans was no more than average, despite there being a degree of administrative decentralization in the forms of de-concentration and delegation used. This survey also showed that, according the management index, these districts did not have the necessary autonomy or sufficient funds of their own to conclude their investment projects, in good part due to the guidelines imposed by the City of Bogotá's Mayor's Office.

KEYWORDs: local budget; intergovernmental relations; regional economic planning.

\section{Introducción}

En Colombia se ha llevado a cabo el modelo de descentralización hacia los departamentos y municipios y es en la Constitución de 1991 donde se profundiza dicho modelo. Adicionalmente, se le da a Bogotá el carácter de "Distrito Capital", permitiéndole gozar de un régimen especial a través del Estatuto Orgánico de la Ciudad —Decreto Ley 1421 de 1993-, con el cual se esperaba desarrollar de manera objetiva el modelo de descentralización.

En este documento se presenta el análisis de la descentralización fiscal y administrativa de las localidades en Bogotá D.C. desde el 2009 hasta el 2012. Para calcular el índice de Desempeño Fiscal se utilizó la información suministrada por la Secretaria Distrital de Hacienda, para el índice de Capacidad Administrativa, la información proporcionada por la Secretaria Distrital de Gobierno y la Veeduría Distrital. Es de anotar que para las localidades de Antonio Nariño y Fontibón no hubo información disponible sobre el cumplimiento de metas para el 2009.

El método utilizado en esta investigación fue el de aplicar la metodología de Evaluación de Desempeño Integral Municipal utilizada por el Departamento Nacional de Planeación (2007), ajustándola a las condiciones y características de la ciudad. Este índice es el resultado del promedio aritmético del índice de Gestión y el índice de Eficacia. Lo anterior, para reconocer a las localidades desde una perspectiva integral al cumplimiento de las metas propuestas en el Plan de Desarrollo Local y en el nivel de capacidad administrativa y fiscal de las mismas. 
En un primer momento, se presenta el marco referencial del proceso de descentralización que ha afrontado la ciudad y el legado que ha dejado el modelo desde el nivel nacional. Posteriormente, se construyen los índices de Gestión, Eficacia y Desempeño integral para analizar la descentralización fiscal y administrativa, exponiendo los resultados obtenidos. Por último, se dan a conocer las conclusiones y algunas recomendaciones.

\section{La descentralización en contexto}

En los territorios donde se aplican estrategias como la descentralización, se le otorga autonomía a los sub-entes para genera un Desarrollo Económico Local (DEL). Así, el propósito es analizar el proceso de descentralización en las localidades de Bogotá, a partir de 2 tipos, fiscal y administrativa, de los 4 que constituyen la descentralización (política, administrativa, fiscal y participativa (Pening, 2003)).

Es importante resaltar que en Bogotá, según Botero y Suarez (2010), lo que existe es un proceso de desconcentración, que de acuerdo con Bernal, Herz, Maydana, Díaz, \& Vega (2008) se da cuando solo son delegadas funciones administrativas a los sub-entes territoriales, mientras que "el término descentralización se puede definir como un proceso a través del cual se realiza una transferencia de recursos (principalmente financieros) y competencias (responsabilidades) desde la administración nacional o central de un Estado hacia las administraciones sub-nacionales" (Junguito, Melo y Misas, 1995:1).

Teóricamente se plantea que dentro del proceso de descentralización, en los sub-entes sometidos al modelo, se genera un alto grado de Desarrollo Económico Local (DEL), dado que "la eficacia de los procesos de descentralización administrativa y política contribuyen, de forma significativa, al surgimiento y expansión de las iniciativas locales" (Vásquez Barquero, 1998:18, citado por Cepal, 2000:76), en busca de descongestionar el poder central y proveer autonomía a los sub-entes. De acuerdo con lo anterior, es importante definir que "un territorio organizado tiene una estructura de administración y, en algunos casos, también de gobierno" (Boisier, 2001:7).

Asimismo, para entender qué se busca con el Desarrollo Económico Local, por un lado Alburquerque (2004:158) afirma que "el desarrollo económico local viene a destacar fundamentalmente los valores territoriales, [...], no sólo en la gran industria, sino en las características generales y locales de un territorio determinado". Por otro lado, Albán y Rendón (2008:62) explican que: "los propósitos del DEL, abarcan lo económico [...], lo sociocultural [...] y lo político [...]”, lo que sustenta los enfoques de la descentralización.

Es necesario resaltar que el éxito de la descentralización se basa en tener fuertes instituciones, como premisa para cumplir los objetivos propuestos. De acuerdo con North (1990), las reglas de juego llevan a la toma de decisiones para ejercer de manera eficiente su funcionalidad, optimizando los recursos otorgados que pueden generar bienestar a la población. Basado en la importancia del institucionalismo, un buen ejercicio del mismo y un conjunto de reglas de juego armonizadas pueden resultar en mejores condiciones de calidad vida y de desarrollo. 
Además, la descentralización persigue unos claros objetivos, que según Baguenard (1994) son,

1) a nivel institucional, se busca que la multiplicación de los centros de decisiones conlleven a una mayor eficacia en la gestión [...]; 2) con la desconcentración del poder se espera que las decisiones sean más acertadas [...]; 3) se busca el pluralismo político, pues se abre la posibilidad a que grupos sociales tradicionalmente excluidos accedan al poder local [...]. [citado por Pening, 2003:125]

Uno de los obstáculos observados que está relacionado directamente con la descentralización y que afecta la gestión administrativa de las localidades es la redistribución del ingreso. De acuerdo con Musgrave y Musgrave (1992), “existen serias restricciones respecto de la capacidad de los gobiernos subnacionales para redistribuir ingresos. [...], la movilidad potencial de los individuos y las empresas, sujetos pasivos de impuestos a la renta, puede impedir la redistribución" (citado por Sánchez, 2003:7).

Esto muestra cómo se ven en dificultades los sub-entes (localidades) para desarrollar el ejercicio de ejecución de los presupuestos dadas las variaciones y limitaciones que se dan al realizar la distribución de los recursos (ingreso).

Cabe aclarar que las localidades no tienen facultades para recaudar tributos, sus ingresos dependen en gran parte de las transferencias realizadas por la administración Distrital. Estos ingresos son utilizados para desarrollar proyectos que nacen de las necesidades de la comunidad, que van ligados al Plan de Desarrollo Local y Distrital y que se encuentran dentro de los gastos administrativos de las alcaldías locales.

\section{Bogotá y sus localidades}

Como estrategia de planificación y en concordancia con el Plan de Ordenamiento Territorial (POT), Bogotá está divida en 20 localidades $^{2}$ y cuenta con 112 Unidades de Planeamiento Zonal (UPZ) ${ }^{3}$ (tabla 1), lo cual facilita los estudios y el desarrollo de los procesos que se llevan a cabo de acuerdo con los propósitos del POT, para mejorar la organización espacial y demográfica de la ciudad.

\footnotetext{
${ }^{2}$ Usaquén, Chapinero, Santa Fe, San Cristóbal, Usme, Tunjuelito, Bosa, Kennedy, Fontibón, Engativá, Suba, Barrios Unidos, Teusaquillo, Los Mártires, Antonio Nariño, Puente Aranda, La Candelaria, Rafael Uribe y Ciudad Bolívar y Sumapaz. En esta investigación no se analiza la localidad de Sumapaz dada su carácter rural.

3 “Áreas urbanas más pequeñas que las localidades y más grandes que el barrio. La función de la UPZ es servir de unidades territoriales o sectores para planificar el desarrollo urbano en el nivel zonal. Son un instrumento de planificación para poder desarrollar una norma urbanística en el nivel de detalle que requiera Bogotá" (Secretaría Distrital de Planeación, s.f.).
} 
Tabla 1

Unidades de Planeamiento Zonal

\begin{tabular}{|lc|}
\hline Localidad & UPZ \\
\hline Usaquén & 9 \\
Kennedy & 12 \\
San Cristóbal & 5 \\
Barrios Unidos & 4 \\
Los Mártires & 2 \\
Antonio Nariño & 2 \\
Puente Aranda & 5 \\
La Candelaria & 1 \\
Rafael Uribe & 5 \\
Ciudad Bolívar & 8 \\
Chapinero & 5 \\
Fontibón & 8 \\
Engativá & 9 \\
Usme & 7 \\
Teusaquillo & 5 \\
Tunjuelito & 2 \\
Bosa & 5 \\
Santa Fe & 5 \\
Suba & 5 \\
\hline & 5 \\
\hline
\end{tabular}

Fuente: Elaboración propia a partir de datos SDP (2011).

Al igual que a nivel nacional, en el Distrito Capital más del 50\% de la población son mujeres (3.919.905). Para Bogotá, las localidades más pobladas son: Kennedy con 1.030.623 habitantes, Suba con 1.069.114 habitantes y Engativá con 851.299 habitantes, las cuales concentran el 39\% de la población total de las localidades. ${ }^{4}$

Los hogares bogotanos están concentrados en los estratos socioeconómicos de la siguiente manera: en Bajo-bajo (Estrato 1) hay el 8.63\%, en Bajo (Estrato 2) el 35.18\%, en Medio-bajo (Estrato 3) el 36.33\%, en Medio (Estrato 4) hay el 12.3\%, en Medio-alto (Estrato 5) el 3.5\% y en Alto (Estrato 6) el 2.66\% del total de las Viviendas.

\footnotetext{
${ }^{4}$ La población total de las 19 localidades referenciadas para este estudio es de 7.525.497 habitantes para el año 2012.
} 
La población de la ciudad capital, durante los últimos años ha percibido altos niveles de pobreza; ${ }^{5}$ a nivel general el porcentaje de población que se consideraba pobre en el 2003 era de $46.1 \%$, en el 2007 de $36.35 \%$ y para el año 2011 de $24.3 \%$. A nivel local en el 2011, el 38.8\% de la población que habita en la localidad de Usme se percibe como pobre, en Santa Fe el 34.1\% y en Ciudad Bolívar el 34\%, al tener una percepción superior a la del promedio Capital.

La intención principal de la creación de las localidades en Bogotá, fue descongestionar el gobierno central de la ciudad, otorgándole facultades a las localidades para llevar a cabo la prestación de servicios y el ejercicio de funciones a cargo del Distrito. Además, con el fin de lograr un adecuado desarrollo de las actividades económicas y sociales que puedan estar cumpliendo las localidades, a partir del Decreto-Ley 1421 de 1993, están las Juntas Administradoras Local (JAL), el Alcalde Local, los Fondos de Desarrollo Local (FDL) y los Consejos Locales de Participación.

La capacidad administrativa de las localidades es la optimización de los recursos disponibles para llevar a cabo los procesos administrativos que la misma demande, estos procesos se realizan mediante los diferentes bienes tangibles o intangibles de los que puedan disponer las localidades. La capacidad para llevar los procesos administrativos depende el cumplimiento de los requerimientos de la ciudadanía y el responder a sus quejas y necesidades de manera oportuna.

Con respecto a los ingresos y gastos de las localidades, de acuerdo con el Decreto 372 de 2010, el sistema presupuestal de las localidades está compuesto por el Presupuesto de los FDL y el Plan Operativo Anual de Inversiones. Igualmente, se señalan los gastos generales de operación y proyectos de inversión clasificados por los objetivos y programas, que mantienen concordancia con el Plan de Inversión establecido por el Plan de Desarrollo Local y Distrital (Secretaría Distrital de Hacienda, 2010:10)

Además, con el Artículo 89 del Decreto 1421 de 1993, se estableció que "mínimo el 10\% de los ingresos corrientes del presupuesto de la administración central del Distrito, se asignará a las localidades teniendo en cuenta las necesidades básicas insatisfechas de la población de cada una de ellas y según índices que para el efecto establezca la entidad de planeación distrital" (Contraloría de Bogota D.C, 2012).

\section{Medición de la descentralización}

La descentralización se experimenta en diferentes formas según se vaya adquiriendo autonomía en la toma de decisiones gubernamentales. ${ }^{6}$ De acuerdo con Pening (2003), la descentra-

\footnotetext{
5 "como un sentimiento subjetivo de los individuos, que se encuentra ligado a los grados de satisfacción que se obtengan de los diferentes "dominios de la vida" (salud, educación, ocio, trabajo, etc.). Por tanto, existen diferentes tipos de pobreza asociados a un determinado dominio. Es decir, una persona puede ser pobre en un dominio específico de la vida" (Aguado Quintero et al., 2007, pág. 9).

${ }^{6}$ Aspectos políticos, administrativos, fiscales y participativos.
} 
lización fiscal podría reconocerse como una autonomía del ente territorial en el momento de implementar nuevas formas de recaudos como impuestos y la reinversión de los mismos. Por su parte, la descentralización administrativa que según Cohen y Peterson (1996), es cuando el gobierno central le transfiere la facultad de la toma de decisiones por parte del ente territorial; "puede ser llevada a cabo en tres modalidades diferentes: Desconcentración, ${ }^{7}$ Delegación ${ }^{8}$ y Devolución"9 (Cohen y Peterson, 1996, citado por Pening, 2003:126).

Otros de los dos tipos de la descentralización son; descentralización política que según Cohen y Peterson (1996), es la facultad de los habitantes del territorio de poder elegir a los gobernantes y que estos mismos generen políticas y las puedan aplicar a la población; y la descentralización participativa que se centra en la participación de los habitantes en la generación de instituciones y la posterior aplicación de las mismas.

El modelo utilizado por el Departamento Nacional de Planeación (2007) para evaluar la descentralización fiscal y administrativa, analiza de manera integral un conjunto de indicadores que relacionan aspectos de gestión, eficacia, eficiencia, ${ }^{10}$ aspectos legales ${ }^{11}$ y de entorno ${ }^{12}$ para los departamentos y municipios, siendo los dos primeros los utilizados para este estudio.

Los índices de Eficacia y Gestión que se utilizan para conocer el desempeño integra de las localidades en Bogotá son definidos por el Departamento de Nacional Planeación (DNP). La Eficacia "mide el desempeño de la entidad para cumplir con las metas propuestas en los planes de desarrollo" (DNP, 2005:19).

A su vez el índice de Gestión

permite determinar la capacidad administrativa; el desempeño financiero de la entidad territorial para dar soporte a sus competencias, a la ejecución de los programas y proyectos del Plan de Desarrollo y a los demás planes estratégicos sectoriales, y la gestión sectorial [...] Por capacidad administrativa se entiende la disposición de recursos humanos, tecnológicos y otros, asignados a dar soporte a los distintos procesos y procedimientos que se cumplen dentro de la organización. [DNP, 2005:22]

\footnotetext{
${ }^{7}$ Se da en una misma organización, con una misma unidad de mando y dirección y puede darse en cualquier nivel del Estado.

${ }^{8}$ Es la ampliación de la desconcentración, adicionando autonomía jurídica y patrimonial.

${ }^{9}$ Es cuando se da autonomía política al ente territorial, pero este tuvo que haber pasado previamente por un proceso de descentralización política.

${ }^{10}$ La eficiencia "evalúa la capacidad que tiene una entidad territorial para maximizar el nivel de producto con los insumos disponibles o para minimizar los insumos para un nivel de producto en los sectores básicos de la gestión local como educación, salud, agua potable, entre otros" (DNP, 2005:21).

11 "Es el cumplimiento legal que se debe llevar a cabo con el fin de responder al seguimiento y a la evaluación de la gestión territorial, conforme a las disposiciones legales que limitan el ámbito de su acción y, por ende, el uso de los recursos transferidos" (DNP, 2005:21).

12 "Busca determinar qué factores de índole político, de orden público o sociodemográficos tienen mayor incidencia en los resultados obtenidos en las evaluaciones de eficacia y eficiencia Para comprender la incidencia del entorno sobre el desempeño es necesario correlacionar los indicadores obtenidos en las evaluaciones de eficacia y de eficiencia con un conjunto de indicadores que reflejan los distintos componentes del entorno" (DNP, 2005:24).
} 
Estos dos índices son los únicos que se ajustan a las localidades de Bogotá, como una forma de adecuar el modelo que implementa el DNP en los departamentos y municipios. En las localidades no se pueden utilizar todos los índices, por los limitantes que éstas tienen en su autonomía; se rescatan algunas funciones que ya tienen como las ejecuciones de presupuesto que siguen los lineamientos establecidos por la Alcaldía Mayor.

\section{Análisis de la descentralización fiscal y administrativa}

La figura 1 resume los índices utilizados para calcular el Índice de Desempeño Integral.

Figura 1

Componentes del Î́ndice de Desempeño Integral

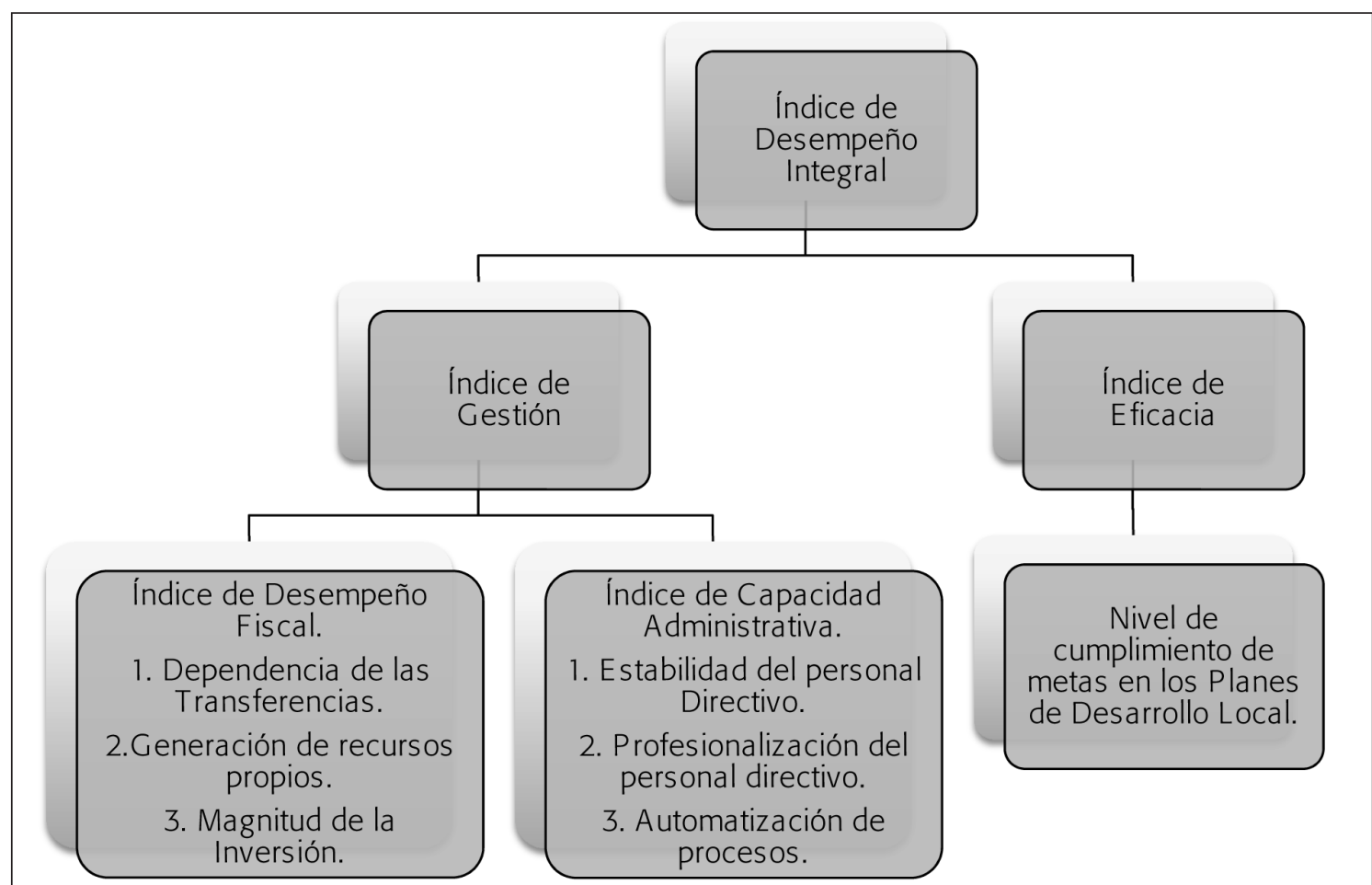

Fuente: Elaboración propia, con base en DNP (2007).

\section{1 Índice de Desempeño Fiscal (IDF)}

El índice de Desempeño Fiscal tiene como objetivo evaluar el estado de las finanzas de la entidad territorial (para este caso de las localidades), y de acuerdo con la metodología utilizada, éste está construido a partir de 6 sub-indicadores financieros. 
Sin embargo, es importante aclarar que teniendo en cuenta las condiciones y características de las localidades y basándose en la información disponible en las ejecuciones presupuestales anuales para cada localidad, la construcción del índice de Desempeño Fiscal para este estudio está compuesta por 3 de los 6 indicadores: Dependencia de las Transferencias, Generación de Recursos Propios y Magnitud de la Inversión.

\subsubsection{Dependencia de las transferencias (DTr)}

Las localidades cuentan con un rubro de transferencias por parte de la administración central. Cabe recordar que el monto a transferir depende de las necesidades básicas y el índice de distribución de recursos que son calculados por la Secretaría Distrital de Planeación año a año.

$$
D T r=\frac{\text { Transferencias de la Admon Distrital }}{\text { Ingresos Totales }} * 100
$$

Dónde un nivel cercano a 100\%, indica que los Fondos de Desarrollo Local (FDL) tiene una mayor dependencia de las transferencias de la Administración Central para la ejecución de los proyectos y metas propuestos en los objetivos estructurales en el Plan de Desarrollo de cada localidad.

\subsubsection{Generación de Recursos Propios (GRP)}

Relaciona la generación de los recursos propios, es decir el peso relativo de los ingresos no tributarios $^{13}$ en el total de los ingresos corrientes. De igual manera ayuda a valorar el esfuerzo que hace las localidades para generar rentas propias (multas, arriendos, fotocopias entre otros) que contribuyen para al financiamiento del gasto.

$$
G R P=\frac{\text { Ingresos No Tributarios }}{\text { Ingresos Corrientes }} * 100
$$

A mayor valor de este indicador, mayor es la capacidad de la localidad de obtener recursos adicionales a las transferencias para el pago de sus gastos de funcionamiento dadas las condiciones de las mismas.

\footnotetext{
${ }^{13}$ Sólo se hace referencia a los ingresos no tributarios como recursos propios debido a que las localidades no tienen la facultad de imponer y recaudar impuestos, facultad que es de la Administración Distrital.
} 


\subsubsection{Magnitud de la Inversión (MInv)}

Cuantifica el nivel de inversión realizado en las localidades con relación al gasto total de las mismas, para el caso de las localidades el gasto de inversión corresponde en su totalidad a la disposición de recursos para llevar a cabo las metas establecidas en los programas de cada uno de los objetivos estructurales en el Plan de Desarrollo Local.

$$
\text { MInv }=\frac{\text { Gasto en Inversión Directa }}{\text { Gasto Total }} * 100
$$

Utilizando el Análisis Factorial por Componentes Principales (AFCP), se tomaron los resultados de la Dependencia de las Transferencias, la Generación de Recursos Propios y la Magnitud de la inversión para cada localidad (i) y se sintetizaron en el Índice de Desempeño Fiscal $\left(\mathrm{IDF}_{\mathrm{i}}\right)$. Esta técnica estadística reduce la información (indicadores) en una dimensión menor, estableciendo ponderaciones $\left(\theta_{\mathrm{i}}\right)$ a cada uno de los indicadores explicativos y perdiendo la menor cantidad posible de información (Tarrádez, 2002). Así,

$$
\mathrm{IDF}_{\mathrm{i}}=\theta_{1 \mathrm{D}} \mathrm{DTr}_{\mathrm{i}}+\theta_{2 \mathrm{G}} \mathrm{GRP}_{\mathrm{i}}+\theta_{3 \mathrm{M}} \mathrm{MInv}_{\mathrm{i}}
$$

De acuerdo con Crocco et al (2006), el AFCP comprende un procedimiento matemático que transforma un conjunto de variables correlacionadas en un conjunto menor o igual de variables no correlacionadas llamadas componentes principales. En este caso, el AFCP transforma el conjunto de los 3 indicadores correlacionados (DTr, GRP y MInv) en un conjunto de 3 componentes principales, $\mathrm{p}_{\mathrm{r}}$, donde $\mathrm{r}=1,2,3$, que varían tanto como sea posible para las 19 localidades.

Para obtener los coeficientes de las combinaciones lineales anteriores, el AFCP hace uso de la matriz de varianzas y covarianzas de los 3 indicadores, DTr, GRP y MInv, la cual se obtuvo con el paquete estadístico SPSS. Esta matriz de varianzas y covarianzas es simétrica, definida no negativa y está dada por:

$$
\Sigma_{\mathrm{i}}=\left[\begin{array}{lll}
\sigma_{\mathrm{iDD}}^{2} & \sigma_{\mathrm{iDG}}^{2} & \sigma_{\mathrm{iDM}}^{2} \\
\sigma_{\mathrm{iGD}}^{2} & \sigma_{\mathrm{iGG}}^{2} & \sigma_{\mathrm{iGM}}^{2} \\
\sigma_{\mathrm{iMD}}^{2} & \sigma_{\mathrm{iMG}}^{2} & \sigma_{\mathrm{iMM}}^{2}
\end{array}\right]
$$

Adicionalmente, el programa SPSS arroja el vector de valores propios $\left(\lambda_{\mathrm{i} 1}, \lambda_{\mathrm{i} 2}\right.$ y $\left.\lambda_{\mathrm{i} 3}\right)$ y la matriz de coeficientes para el cálculo de las puntuaciones en los componentes ( $\alpha_{\mathrm{kiD}}, \alpha_{\mathrm{kiG}}$ y $\alpha_{\text {kim }}$ ), para $\mathrm{k}=1,2$, 3. Efectuando la suma del valor absoluto de los coeficientes de cada componente se obtiene $\mathrm{C}_{\mathrm{ki}}=\left|\alpha_{\mathrm{kiD}}\right|+\left|\alpha_{\mathrm{kig}}\right|+\left|\alpha_{\mathrm{kim}}\right|$, y dividiendo cada uno por la suma $\mathrm{C}_{\mathrm{Ki}}$, asociada a las componentes para recalcular los vectores propios, se tiene que: 


\begin{tabular}{|lccc|}
\hline Indicador & $\mathrm{p} 1$ & $\mathrm{p} 2$ & $\mathrm{p} 3$ \\
\hline DTr & $\alpha_{1 i D}^{*}=\frac{\left|\alpha_{1 i D}\right|}{C_{1 i}}$ & $\alpha_{2 i D}^{*}=\frac{\left|\alpha_{2 i D}\right|}{C_{2 i}}$ & $\alpha_{3 i D}^{*}=\frac{\left|\alpha_{3 i D}\right|}{C_{3 i}}$ \\
GRP & $\alpha_{1 i G}^{*}=\frac{\left|\alpha_{1 i G}\right|}{C_{1 i}}$ & $\alpha_{2 i G}^{*}=\frac{\left|\alpha_{2 i G}\right|}{C_{2 i}}$ & $\alpha_{3 i G}^{*}=\frac{\left|\alpha_{3 i G}\right|}{C_{3 i}}$ \\
& $\alpha_{1 i M}^{*}=\frac{\left|\alpha_{1 i M}\right|}{C_{1 i}}$ & $\alpha_{2 \mathrm{iM}}^{*}=\frac{\left|\alpha_{2 i M}\right|}{C_{2 i}}$ & $\alpha_{3 \mathrm{iM}}^{*}=\frac{\left|\alpha_{3 i M}\right|}{C_{3 i}}$ \\
\hline
\end{tabular}

Por último, el peso final de cada indicador es, entonces:

$$
\begin{gathered}
\theta_{\mathrm{iD}}=\alpha_{1 \mathrm{iD}}^{*} \lambda_{\mathrm{i} 1}+\alpha_{2 \mathrm{iD}}^{*} \lambda_{\mathrm{i} 2}+\alpha_{3 \mathrm{DD}}^{*} \lambda_{\mathrm{i} 3} \\
\theta_{\mathrm{iG}}=\alpha_{1 \mathrm{iG}}^{*} \lambda_{\mathrm{i} 1}+\alpha_{2 \mathrm{iG}}^{*} \lambda_{\mathrm{i} 2}+\alpha_{3 \mathrm{iG}}^{*} \lambda_{\mathrm{i} 3} \\
\theta_{\mathrm{iM}}=\alpha_{1 \mathrm{iM}}^{*} \lambda_{\mathrm{i} 1}+\alpha_{2 \mathrm{iM}}^{*} \lambda_{\mathrm{i} 2}+\alpha_{3 \mathrm{iM}}^{*} \lambda_{\mathrm{i} 3}
\end{gathered}
$$

donde $\theta_{\mathrm{iD}}$ es el peso del indicador DTr, $\theta_{\mathrm{iG}}$ es el peso del indicador, GRP, y $\theta_{\mathrm{iM}}$ es el peso del indicador MInv. Una vez que la suma de los pesos es igual a uno $\left(\theta_{\mathrm{iD}}+\theta_{\mathrm{iG}}+\theta_{\mathrm{iM}}=1\right)$, se puede obtener una combinación lineal de los indicadores para calcular el Índice de Desempeño Fiscal para cada una de las diecinueve localidades $\left(\mathrm{IDF}_{\mathrm{i}}\right)$.

Se aplicó, además, la prueba KMO (Kaiser, Mayer y Olkin), como medida de adecuación muestral, la cual relaciona los coeficientes de correlación de las variables para la técnica de componentes principales y la veracidad para la aplicación de esta técnica en el análisis. El KMO obtenido fue de 0.47 , lo cual implica que la relación entre las variables es baja debilitando la potencialidad de la técnica. Sin embargo, y con el fin de determinar si es posible su utilización, se tomó como referencia la prueba de esfericidad de Bartlett que evalúa la aplicabilidad del análisis factorial de las variables estudiadas.

La Prueba de Esfericidad de Bartlett tiene como hipótesis nula que "se puede aplicar análisis factorial". Si el P-value de dicha prueba es menor a 0.05, se acepta la hipótesis nula.

Como resultado de la prueba KMO y la Prueba de Bartlett, el P-value para esta medición es de $0.021<0.05$, por lo cual se acepta la hipótesis nula y se rechaza la alternativa, sustentando así la utilización de la técnica de análisis factorial para el cálculo del índice de Desempeño Fiscal. Así, se obtuvo las siguientes ponderaciones $\left(\theta_{i}\right)$ para cada uno de los indicadores relacionados. 
Tabla 2

Ponderación de indicadores por componentes principales

\begin{tabular}{|cc|}
\hline Ponderaciones Finales $(\theta)$ & Indicador \\
\hline 0.061 & $\mathrm{DTr}_{\mathrm{i}}$ \\
0.532 & $\mathrm{GRP}_{\mathrm{i}}$ \\
0.407 & $\mathrm{Mlnv}_{\mathrm{i}}$ \\
\hline
\end{tabular}

Fuente: Elaboración propia a partir de los resultados de la técnica de análisis factorial por componentes principales.

A través de la técnica de análisis factorial por componentes principales, se encontró que el Desempeño Fiscal de los FDL es explicado en un $53.2 \%$ por la GRP, en un $40.7 \%$ por la y por último en un $6.1 \%$ por la (Tabla 2), quedando formulado de la siguiente manera:

$$
\mathrm{IDF}_{\mathrm{i}}=0.061 \mathrm{DTr} \mathrm{r}_{\mathrm{i}}+0.532 \mathrm{GRP}_{\mathrm{i}}+0.407 \mathrm{MInv}_{\mathrm{i}}
$$

El indicador sintético resultante de Desempeño Fiscal (IDF), mide globalmente el resultado alcanzado en cada periodo (año) y se encuentra en una escala de 0 a 100. Con base en el resultado del índice se determina el rango de desempeño, para lo cual se tuvo en cuenta los niveles utilizados por la Veeduría Distrital para la medición de nivel de ejecución de metas propuesto en los Planes de Desarrollo Local (tabla 3).

Tabla 3

Nivel de Desempeño

\begin{tabular}{|cc|}
\hline Valor del Índice & Nivel de Desempeño \\
\hline $81 \%-100 \%$ & Bueno \\
$21 \%-80 \%$ & Regular \\
$0 \%-20 \%$ & Malo \\
\hline
\end{tabular}

Fuente: Elaboración propia.

En la tabla 4, se presenta el índice de Desempeño Fiscal promedio de las localidades para el periodo de estudio. Se puede observar que las localidades presentaron un Desempeño Fiscal regular para estos años, mostrando una tendencia decreciente con el pasar del tiempo. En 2011 se presentó el nivel más crítico del indicador promedio para el Desempeño Fiscal, lo cual muestra que la capacidad de las localidades para cubrir sus gastos de funcionamiento con recursos propios no fue posible, dado que con la normatividad vigente estos permanecieron inalterados, y por el contrario, el aumento en la proporción de las transferencias redujo la participación de los recursos propios dentro del total de ingresos corrientes. 
Tabla 4

Índice Promedio de Desempeño Fiscal

\begin{tabular}{|cc|}
\hline Año & Índice Promedio de Desempeño Fiscal \\
\hline 2009 & $30.5 \%$ \\
2010 & $28.0 \%$ \\
2011 & $26.2 \%$ \\
2012 & $27.4 \%$ \\
\hline
\end{tabular}

Fuente: Elaboración propia a partir de los resultados del índice de Desempeño Fiscal.

La localidad de La Candelaria, a pesar de tener un rendimiento decreciente en su desempeño fiscal, mantiene el nivel en promedio más alto de las 19 localidades durante el periodo de estudio. Por su parte, Bosa tuvo un incremento en este índice para el año 2012, no obstante muestra el menor nivel en promedio si se compara con el resto, debido a que la inversión realizada como porcentaje de sus gastos para el año 2011 fue de tan solo el 3.4\% (tabla 5).

Además, basándose en el concepto de descentralización fiscal de Pening (2003), se encontró que las localidades cuentan con bajos recursos disponibles y no tienen la facultad para generar y reinvertir recursos mediante la implementación de impuestos, facultad que solo depende del nivel central. Es claro que para el caso de las localidades no se evidencia una descentralización fiscal, debido al bajo desempeño obtenido en este índice, lo que debilita el objetivo que tiene el Decreto 101 del 2010, en su Artículo 9 "Territorialización de los recursos del Fondo de Desarrollo Local", "el presupuesto financiado con recursos del Fondo de Desarrollo Local, tendrá un anexo que precise, los criterios de distribución territorial de la inversión, de acuerdo con la estructura establecida en el Plan de Desarrollo Local." (Alcaldía Mayor de Bogotá D.C., 2010)

Tabla 5

Índice de Desempeño Fiscal 2009-12

\begin{tabular}{|lllll|}
\hline Localidad & 2009 & 2010 & 2011 & 2012 \\
\hline Antonio Nariño & 0.30 & 0.28 & 0.27 & 0.08 \\
Barrios Unidos & 0.28 & 0.28 & 0.28 & 0.28 \\
Bosa & 0.29 & 0.26 & 0.08 & 0.27 \\
Candelaria & 0.37 & 0.33 & 0.31 & 0.31 \\
Chapinero & 0.33 & 0.29 & 0.10 & 0.28 \\
Ciudad Bolivar & 0.28 & 0.28 & 0.31 & 0.32 \\
Engativá & 0.31 & 0.27 & 0.26 & 0.07 \\
Fontibón & 0.31 & 0.25 & 0.28 & 0.29 \\
\hline
\end{tabular}




\begin{tabular}{|lllll|}
\hline Localidad & 2009 & 2010 & 2011 & 2012 \\
\hline Kennedy & 0.27 & 0.27 & 0.26 & 0.28 \\
Los Mártires & 0.32 & 0.35 & 0.26 & 0.29 \\
Puente Aranda & 0.30 & 0.27 & 0.25 & 0.27 \\
Rafael Uribe & 0.29 & 0.27 & 0.28 & 0.31 \\
San Cristóbal & 0.29 & 0.28 & 0.29 & 0.33 \\
Santa Fe & 0.32 & 0.27 & 0.29 & 0.29 \\
Suba & 0.29 & 0.28 & 0.28 & 0.31 \\
Teusaquillo & 0.33 & 0.28 & 0.31 & 0.29 \\
Tunjuelito & 0.31 & 0.26 & 0.32 & 0.31 \\
Usaquén & 0.32 & 0.28 & 0.29 & 0.33 \\
Usme & 0.31 & 0.28 & 0.26 & 0.28 \\
\hline
\end{tabular}

Fuente: Elaboración propia. Datos Secretaria Distrital de Hacienda.

\section{2 Índice de Capacidad Administrativa (ICA)}

Para la construcción de este índice, se utilizaron los indicadores Estabilidad de personal directivo, Profesionalización de personal directivo y Automatización de procesos, con la información disponible entregada por la Secretaria Distrital de Gobierno para el periodo de estudio. Primero fue necesario hacer la estandarización ${ }^{14}$ de los indicadores ya mencionados y posteriormente se hizo el promedio aritmético.

$I C A=\frac{1}{3}$ Ind.Estabilidad P.D. $+\frac{1}{3}$ Ind.Profesionalizacón P.D. $+\frac{1}{3}$ Ind.Automatización Procesos

El indicador de estabilidad de personal directivo analiza el movimiento de los funcionaros de nivel directivo para la vigencia a evaluar. Cabe aclarar que el Alcalde Local es el personal directivo de libre nombramiento y remoción en las localidades.

Para este indicador se estableció un número base de personal directivo, para todo el periodo de estudio y el número real o total del personal directivo dentro del mismo, para establecer el nivel de permanencia en el cargo. El resultado de este indicador, muestra la probabilidad de permanecer en el cargo directivo.

El indicador de profesionalización del personal directivo mide el grado de formación profesional del personal de la administración, en los niveles directivo y profesional.

\footnotetext{
${ }^{14}$ El método de estandarización de indicadores consiste en convertir los resultados de cada indicador en una escala de 0 a 100, aplicando la formula $\left(\frac{X i}{X \max } * 100\right)$, donde: $\mathrm{X}_{\mathrm{i}}$ : es resultado para cada localidad y Xmax: es el valor máximo del indicador (DNP, 2007).
} 


$$
\text { Índ.Profesionalización }=\frac{\sum \text { Num.Personal Directivo y Profesional, }}{\text { Numero Total de Funcionarios }} * 100
$$

Y, por último, el indicador de automatización de procesos mide si las localidades han implementado aplicativos y/o software en los principales procesos administrativos, que se llevan a cabo en el interior de las localidades.

Como se observa en la tabla 6, las localidades tanto en el año 2009 como en el 2012 presentaron, en promedio, niveles superiores al 90\%, lo que significa alta disposición de recursos humanos, físicos y tecnológicos, que permiten dar soporte a los distintos procesos y políticas que se cumplen en el interior de las localidades. También se puede observar que para el año 2012, el índice de Capacidad Administrativa en todas las localidades fue sobresaliente.

Tabla 6

Índice de Capacidad Administrativa de las Localidades 2009 y 2012

\begin{tabular}{|l|c|c|c|c|}
\hline Localidad & $\mathbf{2 0 0 9}$ & $\mathbf{2 0 1 0}$ & $\mathbf{2 0 1 1}$ & $\mathbf{2 0 1 2}$ \\
\hline Antonio Nariño & 0.91 & 0.89 & 0.91 & 0.91 \\
\hline Barrios Unidos & 0.93 & 0.91 & 0.94 & 0.94 \\
\hline Bosa & 0.92 & 0.91 & 0.85 & 0.91 \\
\hline Candelaria & 0.88 & 0.86 & 0.80 & 0.92 \\
\hline Chapinero & 0.96 & 0.95 & 0.83 & 0.92 \\
\hline Ciudad Bolívar & 0.95 & 0.94 & 0.87 & 1.00 \\
\hline Engativá & 0.94 & 0.91 & 0.83 & 0.91 \\
\hline Fontibón & 0.95 & 0.91 & 0.84 & 0.92 \\
\hline Kennedy & 0.92 & 0.91 & 0.84 & 0.96 \\
\hline Los Mártires & 0.93 & 0.94 & 0.86 & 0.94 \\
\hline Puente Aranda & 0.97 & 0.94 & 0.86 & 0.92 \\
\hline Rafael Uribe Uribe & 0.90 & 0.87 & 0.82 & 0.89 \\
\hline San Cristóbal & 0.97 & 0.93 & 0.86 & 0.93 \\
\hline Santa Fe & 0.95 & 0.93 & 0.82 & 0.92 \\
\hline Suba & 0.94 & 0.93 & 0.85 & 0.93 \\
\hline Teusaquillo & 0.96 & 0.94 & 0.88 & 0.97 \\
\hline Tunjuelito & 1.00 & 1.00 & 0.86 & 0.96 \\
\hline Usaquén & 0.91 & 0.88 & 0.82 & 0.94 \\
\hline Usme & 1.00 & 0.96 & 0.88 & 0.99 \\
\hline
\end{tabular}

Fuente: Elaboración propia. Datos Secretaria Distrital de Gobierno.

Una de las razones por la cual la Capacidad Administrativa presentó niveles mayores al 80\% para el año 2012, es la entrada en vigencia del Acuerdo 308 del 2008: ${ }^{15}$ "[...] Una Bogotá positiva que cuente con unas finanzas sanas y una gestión pública efectiva transparente,

\footnotetext{
${ }^{15}$ Por el cual se adopta el Plan de Desarrollo Económico, Social, Ambiental y de Obras Públicas para Bogotá, D.C., 2008-2012. "BOGOTÁ POSITIVA: PARA VIVIR MEJOR".
} 
abierta a la participación ciudadana y con servicios cercanos a la ciudad." (Alcaldía Mayor de Bogotá D.C., 2010).

En este sentido, y siguiendo a North, se estableció un conjunto de reglas de juego para el mejoramiento de las condiciones de las localidades de Bogotá y el mejoramiento de la calidad de vida de la población.

\section{3 Índice de Gestión (IG)}

El índice de Gestión resume "la capacidad administrativa y financiera de un municipio para materializar los objetivos y metas programadas en el plan de desarrollo local" (DNP, 2007:21). Dentro de la metodología se establece que el cálculo de este índice es el promedio entre el índice de Capacidad Administrativa y el de Desempeño Fiscal.

$$
I G=0.5 I D F+0.5 I C A
$$

Tabla 7

Índice Promedio de Gestión

\begin{tabular}{|cc|}
\hline Año & Índice Promedio de Gestión \\
\hline 2009 & $62 \%$ \\
2010 & $60 \%$ \\
2011 & $56 \%$ \\
2012 & $60 \%$ \\
\hline
\end{tabular}

Fuente: Elaboración propia, a partir de los resultados del Índice de Gestión.

Las localidades en términos de Gestión, presentaron un rango de desempeño superior al 56\% y menor al 80\%. Se puede observar en la tabla 7 que el índice de Gestión disminuyó en 2 puntos porcentuales del 2009 al 2012, y el nivel más bajo se presentó en el año 2011, efecto directamente relacionado con el nivel del desempeño fiscal bajo que hubo para este mismo año.

El resultado del índice de Gestión se deriva de una capacidad administrativa en promedio del 91\%, pero un débil desempeño fiscal del 28\% en promedio, por falta de autonomía. Cabe resaltar las localidades que tuvieron mejor desempeño en el índice de Gestión en el año 2009: Kennedy, Barrios Unidos, Los Mártires y San Cristóbal, quienes presentaron un índice de Gestión superior al promedio (62,3\%). Las localidades de Engativá y Antonio Nariño tuvieron los peores índices para este mismo año. Para el año 2010 quienes se destacaron fueron; Usaquén y Bosa. En el 2011 las localidades de Ciudad Bolívar, Los Mártires, Tunjuelito, Puente Aranda y Santa Fe se destacaron con índices mayores al promedio (55,7\%). En el año 2012, 11 de las 19 localidades estuvieron en niveles mayores al 60\%, resultados superiores al índice promedio de Gestión para este mismo año (figura 2). 
Figura 2

Índice de Gestión de las localidades 2009 y 2012
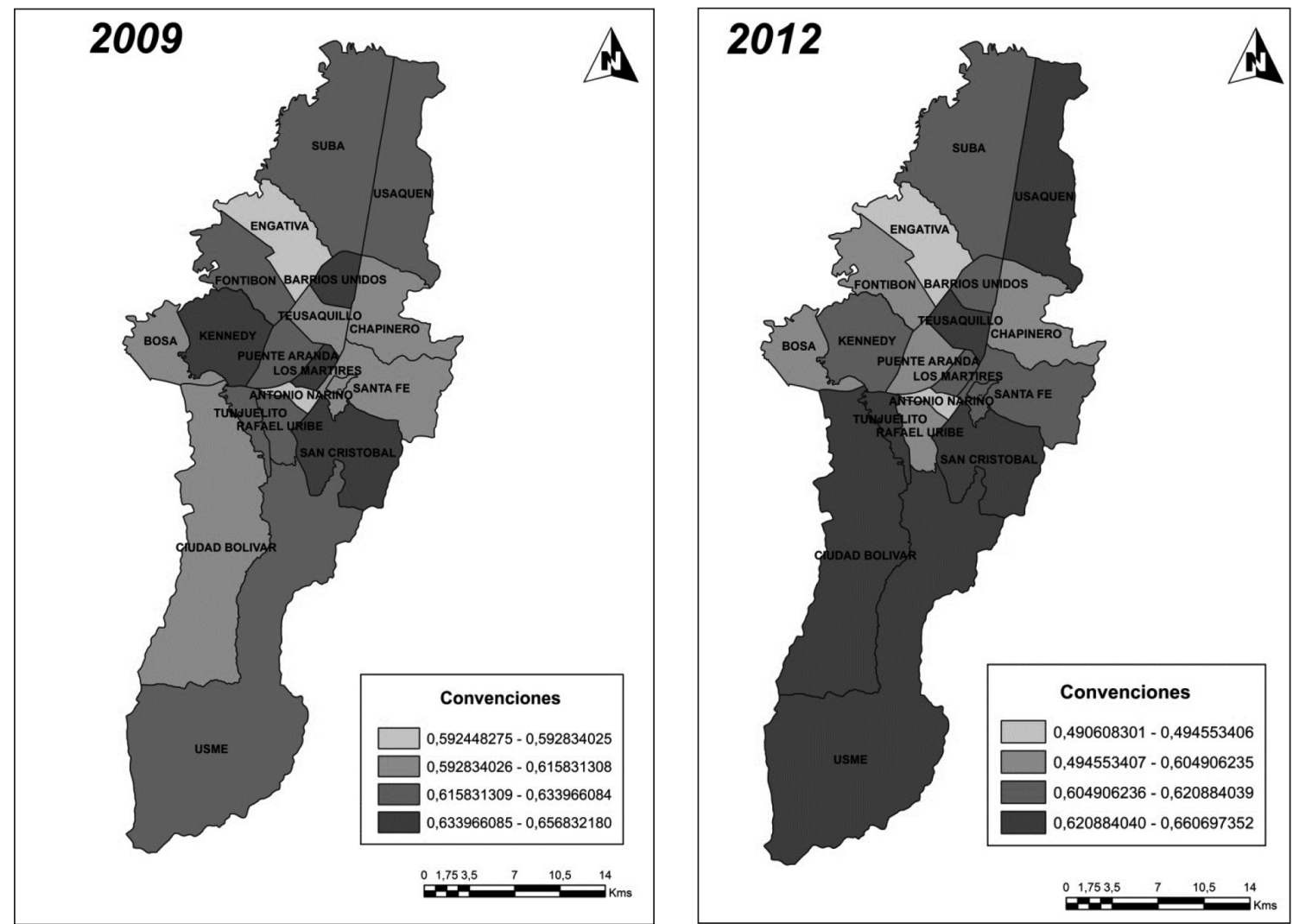

Fuente: Elaboración propia, a partir de resultados del Índice de Gestión.

\section{4 Índice de Eficacia}

El cálculo de este índice, en primer lugar, se establecieron rangos de cumplimiento, dónde niveles de cumplimiento entre el 0\%-20\% presentan un nivel Bajo, entre $21 \%-80 \%$ corresponde a un nivel Medio y entre el $81 \%-100 \%$ a un nivel Alto. Los niveles de cumplimento son tomados de la rendición de cuentas reportada a la Veeduría Distrital, mediante el Acuerdo 131 del 2004. ${ }^{16}$

Para cada rango se contaron el número de metas año a año en cada localidad. Luego se tomaron las frecuencias establecidas por la Veeduría Distrital para estos niveles y así se

\footnotetext{
${ }^{16}$ (Modificado por el Acuerdo 380 del 2009) que obliga a presentar "[...] informes de rendición de cuentas de la gestión contractual y administrativa por parte del Distrito y sus localidades (Entidades Descentralizadas) [...]" (Alcaldía Mayor de Bogotá D.C., 2004).
} 
establecieron las ponderaciones correspondientes a cada uno de ellos, utilizando la técnica de Marca de Clase $^{17}$ que sirve para simplificar el intervalo, obteniendo como resultado el nivel de cumplimiento general para cada localidad.

Utilizando el promedio ponderado de cumplimiento de las metas en los Planes de Desarrollo Local para el periodo 2009-12, que comprende el final del mandato de Samuel Moreno e inicio de Gustavo Petro, se puede observar que a diferencia del Desempeño Fiscal, el índice de Eficacia de las localidades presentó una evolución satisfactoria año tras año; en el 2012 el nivel de cumplimiento promedio fue del 52\% en el total de las metas propuestas, presentando un crecimiento en 10 puntos porcentuales entre el año 2009 y 2012 (tabla 8).

Tabla 8

Índice Promedio de Eficacia

\begin{tabular}{|cc|}
\hline Año & Índice Promedio \\
\hline 2009 & $42 \%$ \\
2010 & $42 \%$ \\
2011 & $48 \%$ \\
2012 & $52 \%$ \\
\hline
\end{tabular}

Fuente: Elaboración propia a partir de resultados datos DNP.

En el figura 2 se puede observar que de acuerdo con los rangos establecidos por la Veeduría Distrital para el año 2009 las localidades presentaron un nivel de cumplimiento de metas medio a nivel general. Las localidades de Los Mártires, Barrios Unidos y San Cristóbal, a diferencia de las otras, fueron las que presentaron mejores niveles de cumplimiento, aunque la consecución de metas estuvo entre el 48\% y el 60\%, esto quiere decir que el Plan de Desarrollo Local se cumplió en alrededor del 50\%. Para el año 2012, las localidades que se destacaron fueron las localidades de Fontibón, La Candelaria, Los Mártires, Suba y Teusaquillo las cuales estuvieron dentro de los niveles del 56\% y 60\%, notándose nuevamente que el avance en el cumplimiento de metas propuesto no supera en mayor magnitud al nivel alcanzado en el año 2009.

Los resultados del índice de Capacidad Administrativa y de Eficacia permiten deducir que las localidades alcanzaron un grado de descentralización administrativa coherente con los recursos físicos, de capital humanos y financieros; aunque cuentan con las transferencias desde la Alcaldía Mayor para el cumplimiento de los objetivos propuestos en los planes de desarrollo, no tienen la autonomía para recaudar recursos propios y como se vio el nivel de eficacia es medio.

\footnotetext{
${ }^{17}$ Es el punto medio de cada intervalo, es decir, el valor que representa todo el intervalo. Se obtiene: Marca de Clase: $\frac{X \min +X \max }{2}$ (Alvarado y Obagi, 2008:19). 


\section{5 Índice de Desempeño Integral}

El Índice de Desempeño Integral, ajustado a las condiciones y características de las localidades en este ejercicio de investigación, es el resultado del promedio aritmético del índice de Gestión y el índice de Eficacia, para evaluar las localidades desde una perspectiva integral, al cumplimiento de las metas propuestas en el Plan de Desarrollo Local y en el nivel de capacidad administrativa y fiscal de las localidades.

En el figura 4, se puede observar que la localidad de Los Mártires presentó el mejor desempeño en los años 2009 y 2012 en lo que concierne al cumplimiento de metas, capacidad administrativa y desempeño fiscal. Las localidades de Engativá, Santa Fe y Tunjuelito, durante los mismos años, tuvieron un desempeño por debajo del 50\%. Finalmente, la localidad de San Cristóbal presentó un deterioro en su Desempeño Integral en comparación con las demás localidades entre el 2009 y 2012.

Figura 3

Índice de Eficacia de las localidades 2009 y 2012
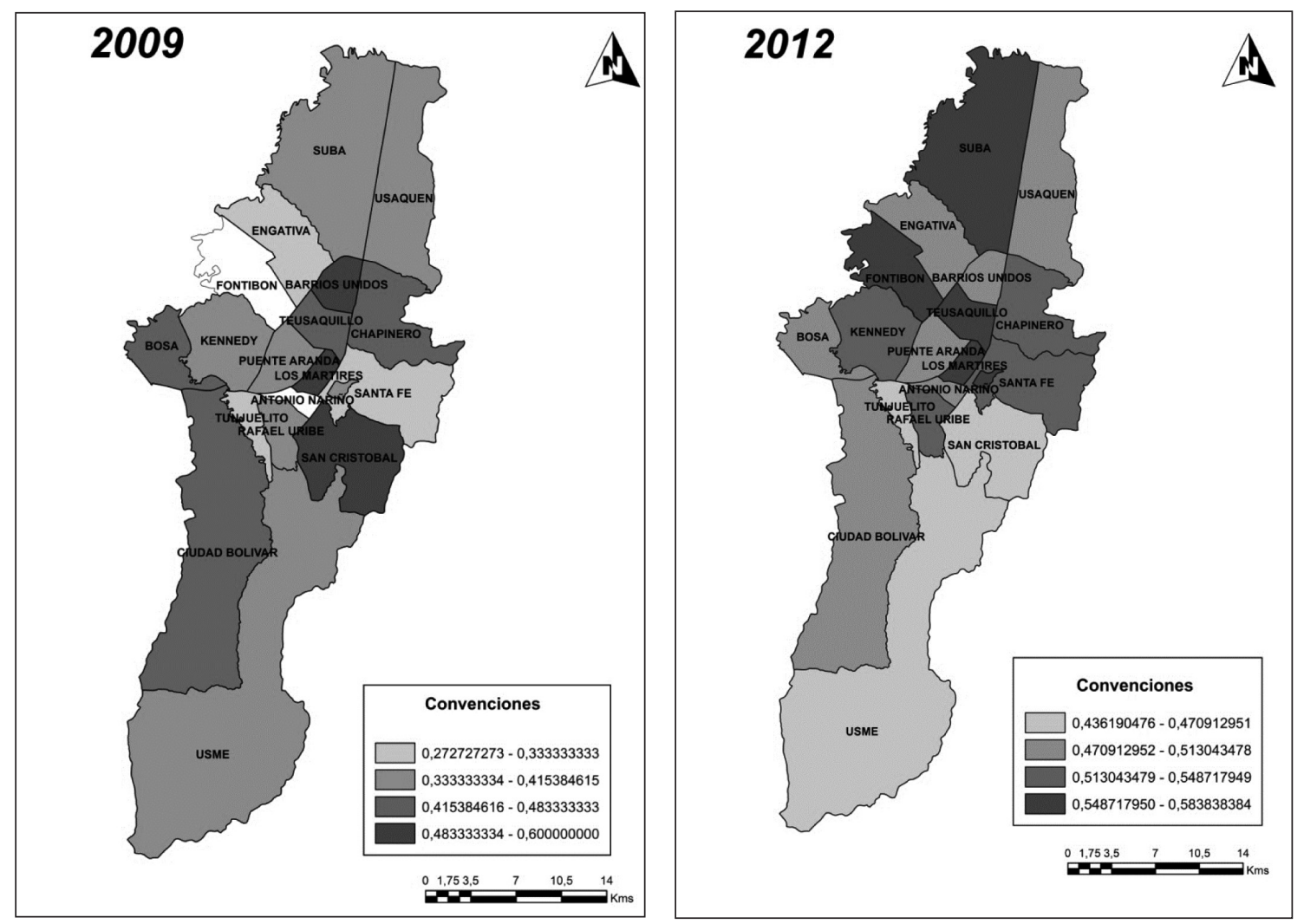

Fuente: Elaboración propia, a partir de resultados del Índice de Eficacia. 
Figura 4

Índice de Desempeño Integral de las localidades 2009 y $2012 z$
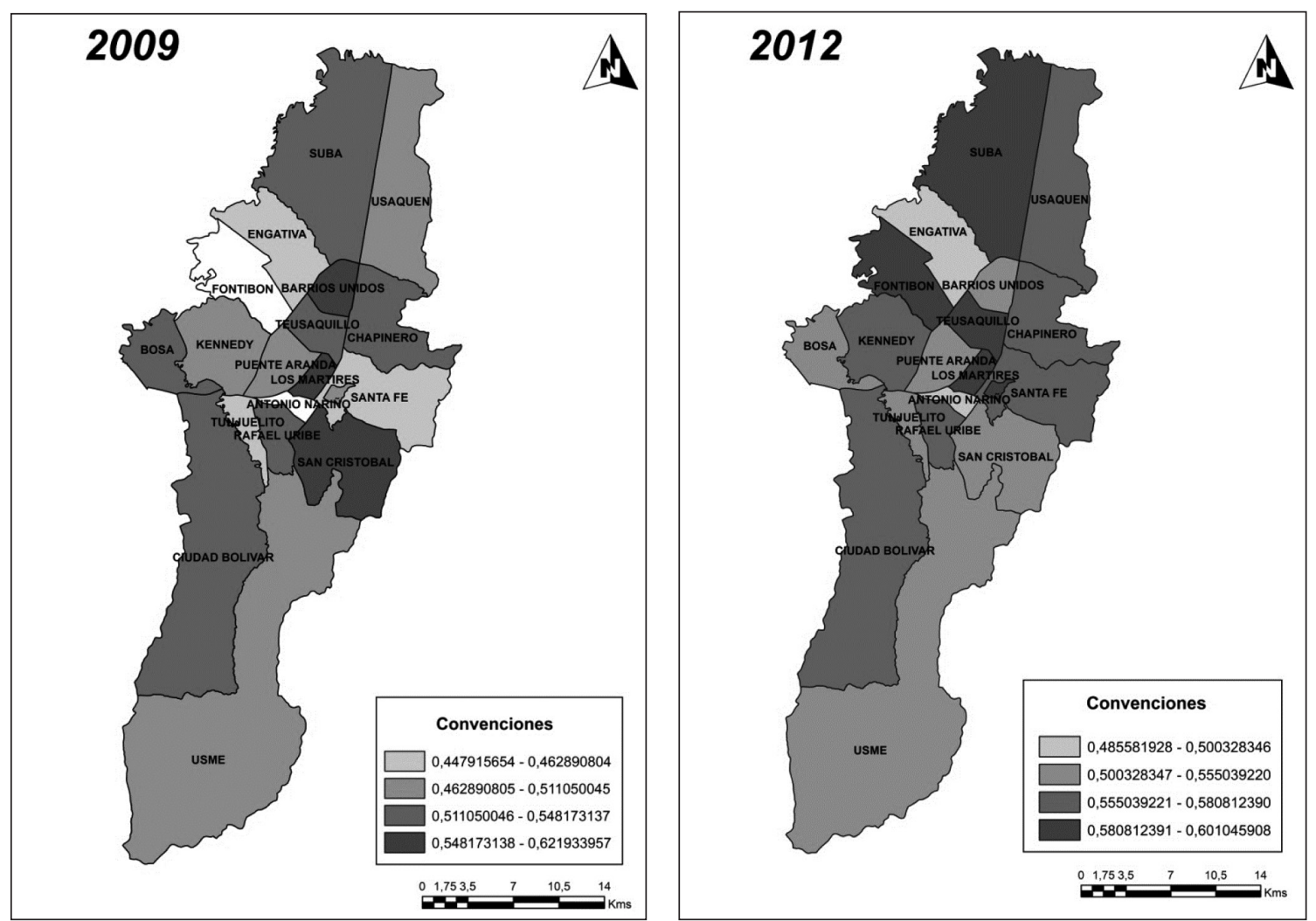

Fuente: Elaboración propia, a partir de resultados del Índice de Desempeño Integral.

\section{Conclusiones}

A partir del análisis de la descentralización fiscal y administrativa realizado en esta investigación a través de la evaluación del Desempeño Integral, se evidenció que las localidades de Bogotá tenían una estructura clara y eficiente para llevar a cabo cada uno de los proyectos propuestos dentro del Plan de Desarrollo Local; y contaban con herramientas como el Presupuesto de los Fondos de Desarrollo Local y el Plan Operativo Anual de Inversiones y con la Secretaria de Hacienda Distrital para el control y revisión de la ejecución de los recursos para cada alcaldía local.

Se encontró que las localidades de Bogotá presentaban un proceso de descentralización administrativa en la que solo se transfirieron competencias para los procesos de contratación pero con dependencia desde la administración central para las decisiones de carácter político. Adicionalmente, eran varias las dificultades para ejecutar los proyectos y metas de los Planes 
de Desarrollo Locales por la carga burocrática existente, lo que obligó a mantener el alto grado de dependencia. Se propone, entonces, que las competencias de cada localidad se amplíen para que le apunten al logro de los objetivos propuestos de una forma más eficiente.

En cuanto a la descentralización fiscal se puede inferir que ha sido de muy baja relevancia en las localidades, ya que éstas no poseen la autonomía para recaudar sus propios ingresos y dependen cien por ciento de las transferencias, lo que limita la ejecución de sus proyectos de inversión. Lo anterior se evidencia con el índice de Eficacia que para el año 2012 logró tan solo un 52\% (en promedio) de cumplimiento de las metas propuestas.

Se propone elaborar una estructura financiera en donde las localidades puedan obtener ingresos propios, por ejemplo, a través de los ingresos provenientes de determinadas multas ocasionadas por incumplimiento de la normatividad existente (mala separación y disposición de desechos, no recoger excremento de perros o maltratar a las mascotas, entre otras), y que ocurran dentro de la misma localidad. En la actualidad, los ciudadanos no cumplen con estas normas por falta de seguimiento y control. La propuesta incentivaría a los alcaldes locales a generar acciones para el acatamiento de las mismas y les proporcionaría recursos adicionales.

Finalmente, con el índice de Desempeños Integral se relacionó tanto el cumplimiento de las metas de los Planes de Desarrollo Locales (índice de Eficacia), como la Capacidad Administrativa y Fiscal (índice de Gestión). El Desempeño Integral de las localidades fue medio y el estudio permitió identificar que localidades como Los Mártires se destacan, mientras que en Tunjuelito y Chapinero su desempeño fue bajo y en algunos años crítico. Para mejorar los resultados del Índice de Desempeño Integral es indispensable profundizar la descentralización fiscal y aumentar el apoyo proveniente de la Alcaldía Mayor en los procesos administrativos.

Para investigaciones posteriores se recomienda realizar un análisis que permita identificar los efectos del proceso de descentralización sobre la calidad de vida de los habitantes. Igualmente, es necesario establecer indicadores adicionales que permitan evaluar este proceso en las localidades del Distrito Capital, de una manera más acorde con las dinámicas de los territorios, utilizando análisis proyectivo de la política pública.

\section{Bibliografía}

AGUADO QUINTERO, Luis F. Q. et al. Midiendo la pobreza a partir de la percepción de los propios individuos: Un calculo para Colombia el Valle del Cauca de la linea de pobreza subjetiva. Revista Atlántica de Economía, v. 6, n. 11, 2007.

ALBÁN, Alvaro M.; RENDÓN, Jorge A. V. Estado del arte de la investigación universitaria en desarrollo económico y local en Colombia. Revista Equidad y Desarrollo, n. 9, p. 61-75, ene./jul. 2008.

ALBURQUERQUE, Francisco. Ll. Desarrollo económico local y descentralización en América Latina. Revista de la Cepal, n. 82, p. 157-171, abr. 2004.

ALCALDÍA MAYOR DE BOGOTÁ D.C. Acuerdo 131. Se establecen informes de rendición de cuentas de la gestión contractual y administrativa. Acuerdo 131 de 2004, 2004. 
ALCALDÍA MAYOR DE BOGOTÁ D.C. Artículo 3. Objetivos del Sistema Presupuestal Local. Decreto 372 del 2010, 2010.

ALCALDÍA MAYOR DE BOGOTÁ D.C. Artículo 9. Territorialización de los recursos del Fondo de Desarrollo Local. Decreto 101 del 2010, 2010.

ALVARADO, Jorge A. V.; OBAGI, Juan J. A. Fundamentos de inferencia estadística. Bogotá D.C.: Pontificia Universidad Javeriana, 2008.

BAGUENERD, Jacques. La déscentralisation. 4. ed. París: Presse Universitaire de France, 1996. (Colección Que sais-je? n. 1879).

BERNAL, Edgar et al. Procesos de descentralización en la Región Andina: Bolivia, Perú, Ecuador y Colombia. In: WENT-INTERNATIONALE WEITERBILDUNG UND ENTWICKLUNG GGMBH (Capacitación y Desarrollo Internacional), 2008. p. 127-145.

BOISIER, Sergio. Desarrollo (local): ¿de qué estamos hablando? Rosario: Homo Sapiens, 2001.

BOTERO, Maria H.; SUÁREZ, Camilo E. Bogotá y la descentralización intraterritorial: crónica de una historia inconclusa. Bogotá: Editorial Universidad del Rosario, 2010.

CEPAL. Comisión Económica para América Latina y el Caribe. Descentralización y desarrollo económico local: una visión general del caso de Colombia. Santiago de Chile: Naciones Unidas, 2000.

COHEN, Jhon M.; PETERSON, Stephen B. Methodological issues in the analysis of descentralization. Cambridge, MA: Harvard Institute for International Development, 1996.

CONSEJO DE BOGOTÁ, D.E BOGOTÁ. Por el cual se crea la Alcaldía Menor "Ciudad Bolívar" y se modifican los límites de las Alcaldías Menores de Tunjuelito y Bosa señalados en el Acuerdo 8 de 1977, 7 de Septiembre de 1983, Acuerdo 14, 1983.

CONTRALORÍA DE BOGOTA D.C. (22 de 8 de 2012). Pronunciamiento respecto de la situacion de ejecución presupuestal de las localidades de la ciudad y la expedicion de la Directiva 5 de 2012, 2012.

CROCCO, Marco A. et al. Metodología de identificação de aglomerações produtivas locais. Nova Economía, v. 16, n. 2, p. 211-241, 2006.

DNP. Departamento Nacional de Planeación. Evaluación del desempeño integral de los municipios 2007. Bogotá: DNP, Dirección de Desarrollo Territorial, 2007.

DNP. Departamento Nacional de Planeación. Metodología para la medición y análisis del desempeño municipal. Bogotá: DNP, 2005.

JUNGUITO, Roberto et al. La descentralización fiscal y la política macroeconómica. Borradores Semanales De Economía, n. 31, p. 1-34, 1995.

NORTH, Douglas. Institutions, Institutional Change and Economic Performance. Cambridge, UK: The Press Syndicate of the University of Cambridge, 1990.

PENING, Jean P. G. Evaluación del proceso de descentralización en Colombia. Economía y Desarrollo, v. 2, n. 1, p. 123-150, 2003. 
PRESIDENCIA DE LA REPÚBLICA DE COLOMBIA. Se otorgan facultades y estructuras administrativas, para las Localidades. Decreto-Ley 1421 de 1993, 1993.

REPÚBLICA DE COLOMBIA. Constitución Política de Colombia. Cap. IV. Art 356, Art 357, Art 358. 1991.

SÁNCHEZ, María del Pilar. Descentralización fiscal y esfuerzo tributario territorial en Colombia. Tesis (maestría publicada) — Universidad Nacional, Bogotá, 2003.

SECRETARÍA DISTRITAL DE HACIENDA. Dirección Distrital de Presupuesto. Manual operativo presupuestal fondos de desarrollo local. Bogotá D.C: Secretaría Distrital de Hacienda, 2010.

SECRETARÍA DISTRITAL DE PLANEACIÓN. Monografías de las Localidades. Bogotá D.C: Secretaría Distrital de Planeación, 2011.

SECRETARÍA DISTRITAL DE PLANEACIÓN. Portal Ordenamiento Territorial. Secretaria Distrital de Planeación. Recuperado el 12 de 02 de 2014, de SDP: <www.sdp.gov.co/portal/page/portal/ PortalSDP/OrdenamientoTerritorial/upzenprocesoderevision/QueEs>, s.f.

TARRÁDEZ, Manuel G. Análisis de componentes principales. Cataluña: Universitat Oberta de Catalunya (UOC), 2002.

María del Pilar Sánchez Muñoz es Candidata a Doctora en Desarrollo Sostenible, Universidad de Manizales (Caldas, Colombia), Magíster en Economía, Universidad Nacional de Colombia (Bogotá, Colombia). Economista, Universidad Católica de Colombia (Bogotá, Colombia). Investigadora del Grupo de Investigación Economía y Desarrollo Humano de la Universidad de La Salle (Bogotá, Colombia). Docente Facultad de Ciencias Contables, Económicas y Administrativas, Universidad de Manizales (Caldas, Colombia).Correo electrónico: madpilarsanchez@gmail.com.

Daniel Fernando Lozano Toscano es Economista de la Universidad de La Salle (Bogotá, Colombia). Investigador Junior del Grupo de Investigación Economía y Desarrollo Humano de la Universidad de La Salle (Bogotá, Colombia). Asesor de proyectos de investigación y lineamientos de Colciencias en políticas públicas para fomentar la Ciencia, Tecnología e Innovación (CT+I) en Colombia para la Facultad de Investigaciones de la Escuela Superior de Administración Pública (Esap) (Bogotá, Colombia). Correo Electrónico: daniellozano16@gmail.com.

Mauricio Fernando Moreno Infante es Economista de la Universidad de La Salle (Bogotá, Colombia). Investigador Junior del Grupo de Investigación Economía y Desarrollo Humano de la Universidad de La Salle (Bogotá, Colombia). Consultor Económico del Departamento Administrativo de Bienestar Social de la Alcaldía Municipal de San José de Cúcuta (Norte de Santander, Colombia). Correo electrónico: mauriciofemoreno@hotmail.com. 